\title{
Relation between Different Types of Treatment and Glycemic Control in Diabetic Pregnant Women
}

\author{
Hakma M. Ageel, Samia A. Hassan*, Fatma M. Ben Rabh, Hiyam Bin Rajab, Nadia S. G. bughalia \\ Department of Pharmacology, Faculty of Medicine, University of Tripoli - Libya
}

\section{Article information}

\section{Article history:}

Received: May, 10, 2021

Accepted: May, 22, 2021

Available online: June, 14, 2021

\section{Keywords:}

Gestational diabetes,

OHA,

Insulin, pregnancy

\section{*Corresponding Author:}

Samia A. Hassan

hassansamia2013@gmail.com

DOI:

https://doi.org/10.53523/ijoirVol8I1ID47

\begin{abstract}
Gestational diabetes mellitus (GDM) is a common medical condition associated with pregnancy. GDM is defined as hyperglycemia that is diagnosed during pregnancy and is not clearly overt diabetes that existed prior to pregnancy. The sample of the study was sixty diabetic pregnant women who came for follow up at obstetrics and gynecology department in TMC in Tripoli medical center during the year 2016. The patients divided according to the type of treatments they received into three groups; A: Patients who took Oral hypoglycemic agents (OHA), B: Patients who took insulin, C: Patents who took both drugs (OHA and insulin), Glycemic control was assessed using HBA1c level, FBS and RBS. This study shows that about $70 \%$ of the patients who took OHA had type 1 diabetes and $30 \%$ had type 2 diabetes. About $77.8 \%$ of patients who took insulin had type 2 diabetes and $22.2 \%$ had type 1 diabetes. And $73.2 \%$ of the patients who were treated with the combination of drugs had type 2 diabetes and $26.8 \%$ had type 1 diabetes. The current study demonstrates that $80 \%$ of patients who took OHA had good control, 33.3\% of patients who took insulin had good control and $53.7 \%$ of patients who took both drugs had good control. Regarding the fasting blood sugar of the patients, the result illustrates the following; High level was reported in $20 \%$ of OHA group, $33.3 \%$ of insulin group and $31.7 \%$ of both drugs group. On the birth weight, the macrosomia $(>4 \mathrm{~kg})$ was more in insulin group $(33.3 \%)$ than other groups $(10 \%$ of OHA and $31.7 \%$ of both drugs group). GDM is a condition that should be treated and prevented, as uncontrolled GDM can affect the mother and the fetal growth.
\end{abstract}

\section{Introduction}

Gestational diabetes mellitus (GDM) is a common medical condition associated with pregnancy. GDM is defined as hyperglycemia that is diagnosed during pregnancy and is not clearly overt diabetes that existed prior to pregnancy [1]. Current research suggests that mothers suffering from GDM have a 50\% chance to develop type 2 diabetes mellitus (T2 DM) in later life following their diagnosis of GDM [2]. Maternal hyperglycemia are more 
likely to increase glucose delivery to the fetus, to cause fetal hyperinsulinemia and increased fetal growth. Therefore, predisposing to birth trauma, increase chance for women to caesarean deliveries, and gives rise to the Intergeneration cycle of diabetics and obesity. Babies born to mother with GDM have many complications include hypoglycemia, hyperbilirubinemia, respiratory distress syndrome, cardiomyopathy, and hypocalcemia [3, 4]. In order to maintain this cycle effective intervention and early management for GDM are required.

Recently in the United States GDM occurs in up to $14 \%$ of all pregnancies, approximately 200,000 cases annually [1]. As well as the prevalence of T2 DM has increased over the past few decades and an increase in the incidence of GDM has also been observed.

Between 1994 and 2002, the incidence of GDM doubled [5]. The rise in GDM can likely be attributed to improved screening and diagnostic tools, as well as to the climbing rate of obesity in the U.S [6]. Excessive caloric consumption and lack of exercise are the major causative factors contributing to obesity. Identifying and aggressively treating GDM may decrease morbidity and mortality in infants [6].

Medical nutrition therapy (MNT) is an exciting new opportunity for GDM treatment and prevention. There are no specific nutritional recommendations since they must be individualized [6]. MNT is best initiated and managed by a registered dietician or skilled individual with experience in GDM management [6]. Additional nonpharmacologic treatments include encouraging for 30 minutes of physical exercise daily for women with no medical or obstetric contraindications $[1,6]$.

After lifestyle intervention, the next-stage of treatment is typically recommended by the ADA insulin injection S.C when there are signs of excessive fetal growth or maternal blood sugar that are not controlled [6]. The ADA defines failure to maintain goals when self-monitoring of blood glucose (SMBG) levels result in fasting plasma glucose $(\mathrm{FPG})>105 \mathrm{mg} / \mathrm{dL}, 1$ hour postprandial $>155 \mathrm{mg} / \mathrm{dL}$, or 2-hour postprandial >130 mg/dL. [American Diabetes Association, 2004] Insulin is the only FDA-approved medication for the treatment of gestational diabetes and has been the mainstay of treatment when MNT is not effective [6, 9].

Insulin: Insulin is the only FDA-approved medication for the treatment of gestational diabetes [3, 9]. Human insulin (Neutral Protamine Hagedorn [NPH] and Regular Insulin) is the least immunogenic of the available insulin brands; however, lispro and aspart insulin develop antibodies at rates and titters comparable to human regular insulin.

The clinical data submitted that Lispro and aspart appears to display efficacy, safety, minimal transfer across the placenta, and no teratogeneic effects. Most studies demonstrate reduced postprandial glucose compared with human regular insulin. Randomized, controlled trials (RCTs) have not been done with glulisine (short-acting insulin) or any of the long-acting insulin. When longer acting agents are needed, NPH insulin can be used as an intermediate-acting insulin [6].

Although glyburide is not FDA approved management for GDM, there is supporting evidence that it can be used as an adjunct to MNT and physical exercise when additional therapy is needed [6,7]. Glyburide is the only sulfonylurea that has reported minimal placenta transfer and has not been associated with excess neonatal hypoglycemia [6].

However, glyburide has been associated with maternal weight gain, preeclampsia, neonatal jaundice and neonatal hypoglycemia [8]. In contrast, metformin has not been combined with prenatal complications [12]. This study aims to compare glycemic control between different types of treatment used in diabetic pregnant women.

\section{Experimental Procedure}

This study was case series study. It was conducted in Tripoli medical center during the year 2016. Sixty diabetic pregnant women who came for follow up at obstetrics and gynecology department in TMC were randomly selected for the study. The patients were divided according to the type of treatment they took into three groups; Group A: Patients who take Oral hypoglycemic agents (OHA), Group B: Patients who take insulin, Group C: Patents who take both drugs (OHA and insulin), Glycemic control was assessed using HBA1c level, FBS and RBS. Statistical 
analysis was computerized using SPSS version 21 that was used for data entry and analysis. All results are presented as frequencies, means \pm standard deviation and percentages. Categorical data were compared using the Chi-square test and Fisher's exact test if appropriate. A P-value of less than or equal to 0.05 was considered statistically significant.

\section{Results and Discussion}

Pregnant women with hyperglycemic associated with gestational or preexisting diabetes mellitus are at risk of adverse maternal and preinatal outcomes. The main goal to glycemic control in pregnant women with diabetes is dietary supplements, with the addition of insulin when diet alone is not adequate. For these reasons, insulin therapy is effective in achieving the suitable levels of glycemia, but it is inconvenient and expensive $[10,11]$ on other hand an alternative approach would be more attractive. Several recent studies advised that sulfonylurea drugs are not to be given during pregnancy because of their potential to cause neonatal hypoglycemia and fetal teratogenic effects [12]. This advice is based mainly on studies done before the availability of new drugs such as glyburide and glipizide, which are in common use today. Laboratory studies have shown that glyburide does not cross the human placenta in significant quantities, on the contrary to older sulfonylurea drugs and metformin. On the basis of these results and the relatively mild hyperglycemia in most pregnant women with gestational diabetes, they recommended that glyburide might be an alternative to insulin therapy in pregnant women with GDM [13].

Insulin is also associated with increased gestational weight gain, requires frequent self-injection and blood glucose monitoring, as well as increases risk of hypoglycemia. However, this treatment is inconvenient and expensive because it requires refrigerated storage and skilled handling, which are not always available in low-income countries.

Some emerging drugs, such as oral hypoglycemic are promising, but long-term effects on the mother and fetus has been controversial because case reports and small sample studies have reported many adverse effects including neonatal hypoglycemia and teratogenicity. Furthermore, several studies with glyburide and metformin reported similar or even better neonatal outcomes if compared to insulin [14].

The purpose of the current study was to compare glycemic control between different types of treatment in diabetic pregnant women. This study shows that most of the patients in each group were between 30 and 40 years. The result also shows that more percentage of older patients were in OHA group than the other groups (insulin and both drugs groups). The result was similar to Tertti and Balani studies in which most of the patients were between 30 and 35 years and patients in the OHA were much older than the insulin group $[14,15]$.

In contrast, the result of Rowan J A reported that patients who took insulin were older than patients who took OHA [16].

The result reported that more patients in the OHA group had better glycemic control than the other groups. This study also showed that patients who took both drugs had better glycemic control than the insulin group. HBA1c level in late pregnancy was better in the OHA group followed by both drugs group than the insulin group. The same result was reported by Balani and Rowan associations that found the mean of HBA1c level was lower in patients who took OHA than patients who took insulin [16].

The result of Langer study was in disagreement with the current study in which the mean HBA1c level was lower in patients who took insulin than in patients who took OHA [13]. However, Tertti and colleagues showed that the mean HBA1c level was the same in patients who took OHA and patients who took insulin [14]. Moreover, the result of Faraci also observed that there was no significant difference in postprandial glucose control between insulin and OHAs [17].

Therefore, with regards to fasting blood sugar, high level was reported in the insulin group. As well as the high random of blood sugar was also higher in the insulin group. This observation suggests that better control was seen in OHA and both drugs group. The result was similar to a published study by Tertti and his colleagues (2008) they 
found lower level of FBS and RBS in the OHA group [14]. However, Rowan study demonstrated that FBS level was the same between OHA and insulin groups while RBS was lower in OHA group [16]. The result of Langer showed that FBS was lower in the OHA group while RBS was lower in the insulin group [13].

Regarding the neonates, the result illustrated that the percentage of macrosomia was higher in the insulin group. Moreover, the result showed that good neonatal condition was seen more in insulin group. Therefore, the result is constant with Tertti and Balani associations studies there were macrosomia and neonatal complications seen more in insulin group $[14,15]$. However, the study of Langer and his colleagues is conflicting with this result which macrosomia is more in OHA but neonatal complications were more in the insulin group [13].

Table (1). Age distribution of the patients.

\begin{tabular}{|lccc|}
\hline 20 - 30 years & OHA & Insulin & Both \\
& & & $10(24.4 \%)$ \\
\hline 30 - 40 years & $5(50 \%)$ & $6(66.7 \%)$ & $23(56.1 \%)$ \\
\hline > 40 years & $3(30 \%)$ & $1(11.1 \%)$ & $8(19.5 \%)$ \\
\hline P value & & 0.883 & \\
\hline
\end{tabular}

Table (2). Type of diabetes distribution, DM Type of treatment.

\begin{tabular}{|lccc|}
\hline & OHA & Insulin & Both \\
\hline Type 1 & $7(70 \%)$ & $2(22.2 \%)$ & $11(26.8 \%)$ \\
\hline Type 2 & $3(30 \%)$ & $7(77.8 \%)$ & $30(73.2 \%)$ \\
\hline P value & \multicolumn{2}{|c}{0.026} & \\
\hline
\end{tabular}

Table (3). HBA1c level in early pregnancy, pre-treatment Type of treatment.

\begin{tabular}{|lclc|}
\hline HBA1c pre treatment & OHA & Insulin & Both \\
\hline Tight Controlled (4-<7) & $8(80.0 \%)$ & $3(33.3 \%)$ & $22(53.7 \%)$ \\
\hline Accepted (7 -<9) & $2(20 \%)$ & $5(55.6 \%)$ & $14(34.1 \%)$ \\
\hline Poor controlled (>9) & $0(0 \%)$ & $1(11.1 \%)$ & $5(12.2 \%)$ \\
\hline P value & 0.301 & & \\
\hline
\end{tabular}

Table (4). HBA1c level in late pregnancy.

\begin{tabular}{|c|c|c|c|}
\hline \multicolumn{4}{|c|}{ HBA1c post treatment } \\
\hline $3-5.5$ & $5(50 \%)$ & $2(22.2 \%)$ & $17(41.5 \%)$ \\
\hline $5.5-7$ & $4(40 \%)$ & $7(77.8 \%)$ & $21(51.2 \%)$ \\
\hline $7-7.51$ & $1(10 \%)$ & $0(0 \%)$ & $3(7.3 \%)$ \\
\hline P value & & 0.533 & \\
\hline
\end{tabular}


Table (5). Fasting blood sugar of the patients.

\begin{tabular}{|llrr|}
\hline FBS & OHA & Insulin & Both \\
\hline Normal & $8(80 \%)$ & $6(66.7 \%)$ & $28(68.3 \%)$ \\
\hline High & $2(20 \%)$ & $3(33.3 \%)$ & $13(31.7 \%)$ \\
\hline P value & \multicolumn{3}{c|}{0.247} \\
\hline
\end{tabular}

Table (6). Random blood sugar of the patients.

\begin{tabular}{|lccc|}
\hline RBS & OHA & Insulin & Both \\
\hline Normal & $7(70 \%)$ & $3(33.3 \%)$ & $20(48.8 \%)$ \\
\hline High & $3(30 \%)$ & $6(66.7 \%)$ & $21(51.2 \%)$ \\
& & & \\
\hline P value & 0.286 & & \\
\hline
\end{tabular}

Table (7). Mode of delivery treatment.

\begin{tabular}{|lcrc|}
\hline Mode of delivery & OHA & Insulin & Both \\
\hline C/S & $6(60 \%)$ & $4(44.4 \%)$ & $25(61 \%)$ \\
\hline NVD & $4(40 \%)$ & $5(55.6 \%)$ & $16(39 \%)$ \\
\hline P value & 0.656 & & \\
\hline
\end{tabular}

Table (8). Birth weight.

\begin{tabular}{|lcrc|}
\hline BW & OHA & Insulin & Both \\
\hline$>\mathbf{4 ~ k g}$ & $1(10 \%)$ & $3(33.3 \%)$ & $13(31.7 \%)$ \\
& & & \\
\hline < $\mathbf{~ k g}$ & $9(90 \%)$ & $6(66.7 \%)$ & $28(68.3 \%)$ \\
\hline P value & 0.369 & & \\
\hline
\end{tabular}

Table (9). Fetal condition.

\begin{tabular}{|lcrc|}
\hline Fetal condition & OHA & Insulin & Both \\
\hline Bad & $3(30 \%)$ & $2(22.2 \%)$ & $17(41.5 \%)$ \\
\hline Good & $7(70 \%)$ & $7(77.8 \%)$ & $24(58.5 \%)$ \\
\hline P value & & & \\
\hline
\end{tabular}

\section{Conclusions and Recommendations}

GDM is a condition that should be treated and prevented, as uncontrolled GDM can affect the mother and the fetal growth. Fortunately, pregnant women with controlled GDM can be effective at managing glycaemia and improve fetal outcomes. As such, long-term health complications can be reduced for both the mother and the child. Furthermore, many of these therapies such as insulin has been used to treat GDM. However, current researches are analyzing the safety and efficacy of oral agents such as glyburide and metformin. Oral agents may offer 
attractive and alternatives ways to patients with GDM. There is a need for further studies, both in animals and humans, to support the evidence, and ensure the safety of both the mother and the fetal.

\section{References}

[1] American Diabetes Association. Standards of medical care in diabetes. Diabetes Care. (2009); 32:S13-S61.

[2] Herath H., Herath R. and Wickremasinghe R. Gestational diabetes mellitus and risk of type 2 diabetes 10 years after the index pregnancy in Sri Lankan women-A community based retrospective cohort study. PLoS One. (2017); 12(6).

[3] Perkins J.M., Dunn J.P. and Jagasia S.M. Perspectives in gestational diabetes mellitus: a review of screening, diagnosis, and treatment. Clinical Diabetes. (2007); 25(2):57-62.

[4] Ramanathan J. and Ivester T. Diabetes mellitus in pregnancy: pathophysiology and obstetric and anesthetic management. SeminAnesth. (2002); 21(1):26-34.

[5]Dabelea D., Snell-Bergeon J.K., Hartsfield C.L., Bischoff K.J., Hamman R.F., McDuffie R.S., and Kaiser Permanente of Colorado (GDM) Screening Program. Increasing prevalence of gestational diabetes mellitus (GDM) over time and by birth cohort: Kaiser Permanente of Colorado (GDM) Screening Program. Diabetes Care. (2005); 28:579-584.

[6] Metzger B.E., Buchanan T.A., Cosutan D.R., de Leiva A., Dubger D.B., Hadden D.R., Hod M., Kitzmiller J.L., Kjos S.L., Oats J.N., Pettitt D.J., Sacks D.A. and Zoupas C., Summary and recommendations of the Fifth International Workshop-Conference on Gestational Diabetes Mellitus. Diabetes Care. (2007); 30(2): S251-260.

[7] Langer O., Conway D.L., Berkus M.D., Xenakis E. M. and Gonzales O. A comparison of glyburide and insulin in women with gestational diabetes. N Engl J Med. (2000); 343:1134-1138.

[8] Simmons D. Safety considerations with pharmacological treatment of gestational diabetes mellitus. Drug Saf. (2015); 38, 65-78.

[9]Carr D.B. and Gabbe S. Gestational diabetes; detection, management, and implications. Clinical Diabetes. (1998); 16(1):4-11.

[10] Farrar D. hyperglycemia in pregnancy: prevalence, impact, and management challenges. Int J Womens Health. (2016); 8: 519-527.

[11] Kishimoto M., Kato R. and Oshiba Y.M. Impact of dietary counseling on the perception of diet in patients with gestational diabetes mellitus. Diabetol Int. (2020);12(2): 151-160.

[12] Langer O., Deborah L., Conway D.L., Berkus M.D., Xenakis M.D., and Gonzales, O.R. A Comparison of Glyburide and Insulin in Women with Gestational Diabetes Mellitus. N Engl J Med; (2000); 343:1134-1138.

[13] Langer O., Conway D., Berkus M., Xenakis E. and Gonzales O. A. Comparison of Glyburide and Insulin in Women With Gestational Diabetes Mellitus. Obstetric and Gynecologic Survey. (2001); 56(3):126-127.

[14] Tertti K., Ekblad U., Vahlberg T. and Rönnemaa T. Comparison of Metformin and Insulin in the Treatment of Gestational Diabetes: A Retrospective, Case-Control Study. The Review of Diabetic Studies: RDS. (2008); 5(2):95-101.

[15] Balani J., Hyer S., Rodin D., Shehata H. Pregnancy outcomes in women with gestational diabetes treated with metformin or insulin: a case-control study. Diabetic Medicine. (2009); 26(8):798-802.

[16] Rowan J., Hague W., Gao W., Battin M., Moore M. Metformin Versus Insulin for the Treatment of Gestational Diabetes. Obstetrical \& Gynecological Survey. (2008); 63(10):616-618.

[17] Faraci M, Di Prima FAF, Valenti O., Hyseni E., Monte S., Giorgio E. and De Domenico R. Treatment of gestational diabetes: oral hypoglycemic agents or insulin? Journal of Prenatal Medicine. (2011); 5(3):63-64. 